\title{
Design of PSS and TCSC Damping Controller Using Particle Swarm Optimization
}

\author{
Maxwell Martins de Menezes $^{1}$ - Percival Bueno de Araujo ${ }^{2}$. \\ Danilo Basseto do Valle ${ }^{3}$
}

Received: 9 November 2015 / Revised: 6 June 2016 / Accepted: 20 June 2016 / Published online: 11 July 2016

(C) Brazilian Society for Automatics-SBA 2016

\begin{abstract}
In this paper, we propose a methodology to tune power system stabilizers and thyristor-controlled series capacitor damping controllers simultaneously. The particle swarm optimization algorithm is incorporated into a power system model to tune the parameters of supplementary damping controllers. A test power system of 10 generators, 39 buses and 46 transmission lines is simulated to validate the use of this optimization algorithm. The tuning of supplementary damping controllers using the proposed methodology increases their performance to provide additional damping to low-frequency oscillation modes in the simulated power system. The controller position is determined by the participation factors (power system stabilizers) and the distance between the interest pole and the zero of the open-loop transfer function of the power oscillation damping controller (thyristor-controlled series capacitor-power oscillation damping). The results show the operating efficiency of the power system after using the optimization technique to tune damping parameters, thereby improving power system integrity. The power sensitivity model is used for the simulations presented in this work focusing on the analysis small-signal stability.
\end{abstract}

Maxwell Martins de Menezes

maxwell.menezes@unoesc.edu.br

Percival Bueno de Araujo

percival@dee.feis.unesp.br

Danilo Basseto do Valle

danilodovalle@gmail.com

1 Universidade do Oeste de Santa Catarina, Rua Getúlio Vargas, 2125, Joaçaba, SC, Brazil

2 Universidade Estadual Paulista, Av. Professor José Carlos Rossi, 1370, Ilha Solteira, SP, Brazil

3 Instituto Federal de São Paulo (IFSP), Avenida do Universitário, 145, Tupã, SP, Brazil
Keywords Small-signal stability · TCSC · Damping controllers · PSO

\section{Introduction}

Interconnections of power systems may result in insufficient damping of low-frequency oscillations. These variations trigger a series of events that can result in inadequate and even critical operating conditions. Generally, oscillations are classified as local, inter-area or intra-plant modes and are classified according to their natural undamped frequency (Milano 2010; Rogers 2000).

Power system stabilizers (PSSs) (Milano 2010; Talaq 2012) are often used in the control loop of the excitation system of synchronous generators to guarantee damping to low-frequency oscillations, in particular of local oscillation modes. Their impact on inter-area oscillation modes can be ineffective in some power system structures, thus negatively contributing to the damping of other oscillation modes. This situation is even worse when long transmission lines are used for interconnections between areas. Flexible alternating current system (FACTS) (Zhang et al. 2006; Sen and Sen 2009) is commonly used in the electrical energy market because it improves transmission and distribution. A thyristor-controlled series capacitor device (TCSC) was used in the design of this work (Sen and Sen 2009; Meikandasivam et al. 2010). It is suggested that the TCSC should be combined with a power oscillation damping (POD) controller (IEEE STANDARDS 2009; Morsali et al. 2013; Simoes et al. 2009), as this combination is able to introduce additional damping to oscillation modes, especially for inter-area oscillation modes.

The performance of PSSs and TCSC-POD controllers in power systems can cause undesirable oscillations when the 
supplementary controllers are adjusted incoherently or, as happens in some cases, if the controllers are adjusted individually (Jabr et al. 2010). Depending upon the number of supplementary damping controllers, algorithms based on classic techniques such as the decentralized modal control (Araujo and Zaneta 2001) have complicated computational responses in terms of programming and convergence.

The ability to perform a systematic search independent of the number of supplementary controllers was decisive in the choice of the particle swarm optimization (PSO) algorithm for this study (Abido 2002; Bratton and Kennedy 2007). PSO is an optimization algorithm used in various areas, and it can be adapted as a tool to adjust damping controlling parameters (Morsali et al. 2013; Shayeghi et al. 2010; Mahapatra and Jha 2012).

Due to the recent expansion of the power system, research on operation and control alternatives using simulations to analyze small-signal stability is necessary to ensure safety. Hence, it is suggested that local and inter-area oscillation modes are studied after the installation of PSSs and TCSCPOD controllers in a power system. To validate the technique proposed by this work, simulations are presented using a test power system with 10 generators, 39 buses and 46 transmission lines.

This work differs from the others in the literature as the modeling of the devices uses the power sensitivity model, incorporating the PSO algorithm to simultaneously tune $n$ damping controllers (PSS or POD). It is important to remember that the PSO algorithm is completely independent of the number and type of controllers, thus providing a parameter tuning tool for other power systems.

\section{Power System Model}

The models used to represent the power system, including linearized models of damping controllers, are described in this section. These supplementary controllers contribute by damping oscillation modes. In this work, the damping to local oscillation modes is achieved using PSSs, while the TCSCPOD controller enhances damping of inter-area oscillation modes.

A multi-machine system composed of $n$ generators and $n$ buses is considered to represent the power system in the power sensitivity model (PSM) as shown in Eq. (1) where $\Delta x$ is the vector of state variables, $\Delta z$ the algebraic variables and $\Delta u$ the input variables.

$$
\left[\begin{array}{c}
\Delta \dot{x} \\
0
\end{array}\right]=\left[\begin{array}{ll}
J_{1} & J_{2} \\
J_{3} & J_{4}
\end{array}\right]\left[\begin{array}{l}
\Delta x \\
\Delta z
\end{array}\right]+\left[\begin{array}{l}
B_{1} \\
B_{2}
\end{array}\right][\Delta u]
$$

In Eq. (1), $J_{1}$ is a matrix that associates state variables with state variables, and $J_{2}$ is a matrix that correlates state

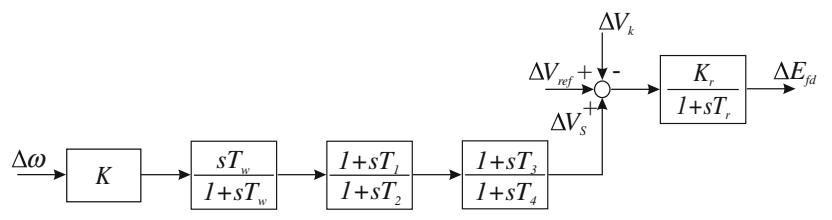

Fig. 1 Representation with AVR and PSS

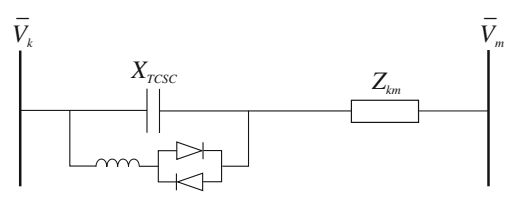

Fig. 2 Circuit equivalent of the TCSC device

variables with algebraic variables. The matrix $J_{3}$ correlates algebraic variables with state variables, and the relationships between algebraic variables with algebraic variables are defined by the matrix $J_{4}$. The input matrices of the system are $B_{1}$ and $B_{2}$.

On considering the elimination of the vector of algebraic variables $\Delta z$, it is possible to represent the power system in the form of state space [Eq. (2)], where $\mathcal{A}$ is the matrix of states and $\mathcal{B}$ is the matrix of inputs.

$$
\begin{aligned}
& \Delta \dot{x}=\mathcal{A} \Delta x+\mathcal{B} \Delta u \\
& \mathcal{A}=J_{1}-J_{2} J_{4}^{-1} J_{3} \\
& \mathcal{B}=B_{1}-J_{2} J_{4}^{-1} B_{2}
\end{aligned}
$$

The model to represent PSSs is illustrated in Fig. 1 (Hassan et al. 2013; Basler and Schaefer 2008). The voltage variation $\Delta V_{s}$ is defined as the resulting output signal, which is directly applied to the control loop of the automatic voltage regulator (AVR) (Basler and Schaefer 2008; Gurrala and Sen 2010).

This controller has a gain $K$ that regulates the amount of damping, a washout block defined by the time constant $T_{w}$ and time constants $T_{1}, T_{2}, T_{3}$ and $T_{4}$ responsible for the required phase compensation (Basler and Schaefer 2008), with the input signal being the variations in the angular velocity of the rotor $\Delta \omega$ of the synchronous generator.

The TCSC can be represented by thyristor-controlled reactors in parallel with a variable capacitive reactance, $X_{T C S C}$ controlled by the firing angle of the thyristors (Zhang et al. 2006). Its model is shown in Fig. 2, with its use causing a decrease in the initial reactance, $X_{k m}$, of the transmission line.

The POD controller is used in conjunction with the TCSC device (IEEE STANDARDS 2009) to damp low-frequency oscillations of the power system. Its structure is similar to the PSS, but differs in its operation and its input signal. The basic structure of the TCSC-POD controller is shown in Fig. 3 (Simoes et al. 2009; Shayeghi et al. 2010) with a time con- 


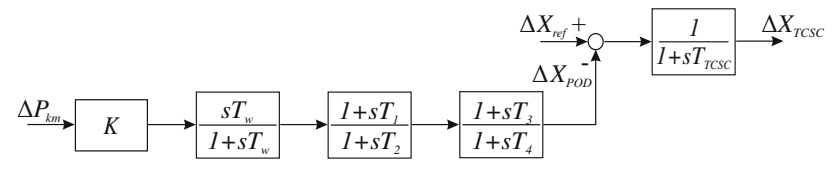

Fig. 3 Block diagram for the TCSC-POD model

stant $T_{T C S C}$ and the signal $\Delta X_{\text {ref }}$ in respect of the reactance deviation of the TCSC in continuous operation.

Additional damping to low-frequency oscillations in the power system is expected with the installation of PSSs and the TCSC-POD controller. For this, the parameters of the PSSs and TCSC-POD controller must be correctly adjusted. The next section describes the PSO algorithm, which is used to tune the parameters of the PSSs and TCSC-POD controller simultaneously.

\section{Particle Swarm Optimization}

The purpose of this study is to adjust the parameters of the PSSs and the TCSC-POD controller simultaneously. In this work, we used the PSO algorithm (Shayeghi et al. 2010; Mahapatra and Jha 2012), which is based on the concept of the movement of several particles in a specific space, provided that they do not approach each other, thereby avoiding collisions, and follow the remaining particles in an orderly manner (Kennedy and Eberhart 1995).

As stated in Shayeghi et al. (2010), the location of the PSS controllers is defined by factors of participation. The damping controller parameters are calculated using the PSO algorithm. Thus, the significant difference is related to the number of controllers tuned simultaneously, the objective function used by the algorithm and the model used to represent the power system. In this work, representations of the devices and controllers as well as the use of the PSO algorithm to tune the parameters that control the POD were incorporated in the power sensitivity model. A key point of this work is the association of a specific eigenvalue in relation to a damping controller, which is routine when using the PSO algorithm. Without this routine, the algorithm would not identify the eigenvalues of interest to confirm the objective function of the algorithm.

The movement of a particle in any space is determined by its speed [Eq. (5)], which varies at each change of position in the specific space. The velocity $v_{i}(t+1)$ and the new position of the particle $x_{i}(t+1)$ from its current position $x_{i}(t)$ are defined in Eqs. (5) and (6), where $i$ is the particle, $t$ is the transition number and $W$ is the search factor (weighted by the current velocity of the particle). The variables $c_{1}$ and $c_{2}$ are weights, and $r_{1}$ and $r_{2}$ are random distributions between 0 and 1.

$v_{i}(t+1)=W v_{i}(t)+c_{1} r_{1} c o_{i}(t)+c_{2} r_{2} s o_{i}(t)$ $x_{i}(t+1)=x_{i}(t)+v_{i}(t+1)$

The velocity of the movement of each particle depends on social $\left(s o_{i}\right)$ and cognitive factors $\left(c o_{i}\right)$ as defined in Eqs. (7) and (8), respectively. These factors are classified according to the positions travelled by the particles. The relationship of each of the particles with its respective best position (Pbest $\left.t_{i}\right)$ is called its cognitive factor, and the relationship of each of the particles with the best position of all the particles (Gbest) is called its social factor.

$c o_{i}=$ Pbest $_{i}-x_{i}(t)$

$s o_{i}=$ Gbest $-x_{i}(t)$

For the displacement of a particle in any space, it is necessary to control the velocity and position. Restrictions for speed and position are defined, in Eqs. (9) and (10), respectively. The constants $v_{\max }$ (maximum speed of a particle of the algorithm), $x_{\min }$ (minimum allowable value for particles) and $x_{\max }$ (maximum allowable value for particles) are defined by the PSO algorithm. These constants limit the search area of the algorithm.

$$
\begin{aligned}
& \left\{\begin{array}{cc}
\text { if } & -v_{\max } \leq v_{i}(t+1) \leq v_{\max }: v_{i}(t+1) \\
\text { if } & v_{i}(t+1)<-v_{\max }:-v_{\max } \\
\text { if } & v_{i}(t+1)>v_{\max }: v_{\max }
\end{array}\right. \\
& \left\{\begin{array}{cc}
\text { if } & x_{\min } \leq x_{i}(t)+v_{i}(t+1) \leq x_{\max }: \\
& x_{i}(t)+v_{i}(t+1) \\
\text { if } & x_{i}(t)+v_{i}(t+1)<x_{\min }: x_{\min } \\
\text { if } & x_{i}(t)+v_{i}(t+1)>x_{\max }: x_{\max }
\end{array}\right.
\end{aligned}
$$

The restrictions imposed by Eqs. (9) and (10) guarantee that the particles do not extrapolate the boundaries of the search area as determined in the design. In the case of this work, the limits are defined by constants calculated by the residue method (Zhang et al. 2012).

To set the damping constant of each controller in the power system, it is necessary to define an objective function [Eq. (11)]. This equation tends to minimize the distance between the calculated oscillation modes of interest $\left(\lambda_{c_{k}}\right)$ and desired oscillation modes of interest $\left(\lambda_{d_{k}}\right)$. $N_{p}$ is the number of eigenvalues of interest related to the damping controllers.

$\min \sum_{k=1}^{N_{p}}\left|\lambda_{c_{k}}-\lambda_{d_{k}}\right|$

Using Eq. (11), the equivalent value of the desired oscillatory mode $\lambda_{d_{k}}$ is calculated for each oscillatory mode of interest from the definition of the desired damping coefficient $\xi_{d_{k}}$ (Kundur 1994). The calculated oscillatory modes $\lambda_{c_{k}}$ are obtained from power system simulations with the parameters of the damping controllers (values of the constants $T_{1}$, 
$T_{2}, T_{3}, T_{4}$ and $K$ ) obtained by means of iterations of the PSO algorithm.

$\lambda_{d_{k}}=-\xi_{d_{k}}\left|\lambda_{d_{k}}\right|+j\left|\lambda_{d_{k}}\right| \sqrt{1-\xi_{d_{k}}^{2}}$

Equation (11) is subject to the set of constraints defined in Eq. (13), with $\lambda_{m}$ being the eigenvalues of matrix $\mathcal{A}$ and $k$ representing the oscillation mode related to the damping controllers. With these restrictions, infeasible solutions will not be allowed; examples of this are the saturation of the modes of damping controllers and eigenvalues with a positive real part.

$$
\begin{aligned}
& K^{\min } \leq K \leq K^{\max } \\
& T_{1}^{\min } \leq T_{1} \leq T_{1}^{\max } \\
& T_{2}^{\min } \leq T_{2} \leq T_{2}^{\max } \\
& T_{3}^{\min } \leq T_{3} \leq T_{3}^{\max } \\
& T_{4}^{\min } \leq T_{4} \leq T_{4}^{\max } \\
& \xi_{d_{k}}^{\min } \leq \xi_{d_{k}} \leq \xi_{d_{k}}^{\max } \\
& \lambda_{m}=\operatorname{Re}\left(\lambda_{m}\right) \pm j \operatorname{Im}\left(\lambda_{m}\right), \forall \operatorname{Re}\left(\lambda_{m}\right)<0
\end{aligned}
$$

From Figs. 1 and 3, it can be seen that the dynamic models relating to the PSSs and the POD controller are similar. They have a gain $K$, a washout block $T_{w}$ and phase lead-lag constants $\left(T_{1}, T_{2}, T_{3}\right.$ and $\left.T_{4}\right)$.

The number of variables to be optimized increases the convergence time of the PSO algorithm. This is compounded with the increase in damping controllers installed in the power system, contributing to define that $T_{w}=10, T_{1}=T_{3}$ and $T_{2}=T_{4}$.

Each particle contains a configuration with the values of the constants $T_{1}, T_{2}$ and $K$ of $\mathrm{n}$ damping controllers installed in the power system. Twenty-five particles are considered, which results in a set of 75 solutions to be controlled by the PSO algorithm for each damping controller inserted in the power system.

With the parameters determined by the PSO algorithm, it is desired that the oscillation modes related to the PSSs and TCSC-POD controller are positioned in a particular region of the complex plane. Figure 4 shows the best regions for local and inter-area oscillation modes.

In Fig. 4, the regions are defined by means of the damping coefficients specified for each oscillation mode. The desired minimum is set as $\xi_{\text {local }}=5 \%$ for local modes and $\xi_{\text {interárea }}=10 \%$ for inter-area modes. The choice of a smaller damping coefficient for local modes is justified because they are not sensitive to changes in the power system operating conditions (Molina et al. 2013).

\section{Results}

The simulated test power system has 10 generators, 39 buses and 46 transmission lines. Known in the literature as the

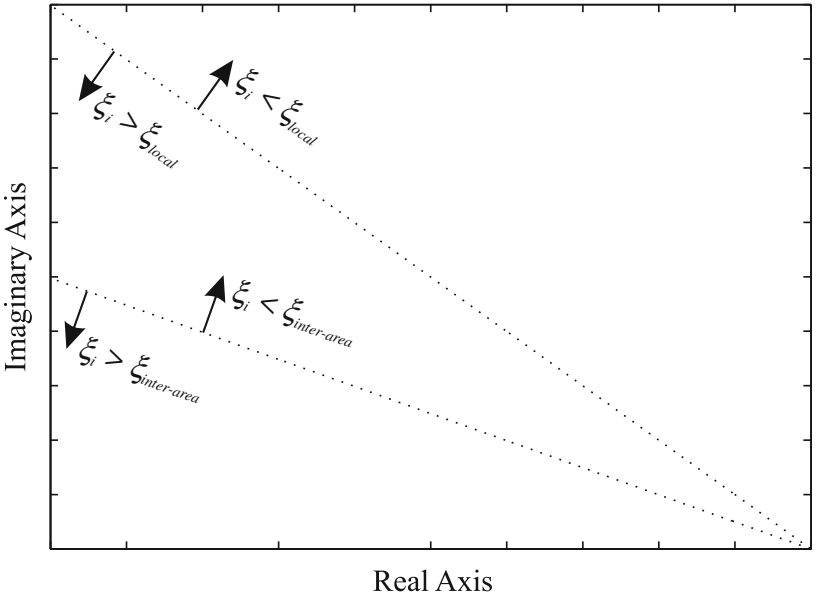

Fig. 4 Region of desired concentration for the eigenvalues

"New England," this system has local and inter-area oscillation modes with low and even unstable damping using the loading data (Araujo and Zaneta 2001). The representative line diagram is shown in Fig. 5.

Using simulations, the behavior of the power system was evaluated by the dominant eigenvalues, damping coefficients $(\xi)$ and the natural undamped frequencies $\left(\omega_{n}\right)$. These results are given in Table 1.

On analyzing the data given in Table 1, eight local oscillation modes and one inter-area oscillation mode, renowned for their natural undamped frequencies $\left(\omega_{n}\right)$, are identified in the test system (Kundur 1994). Modes 1, 5 and 8 (local) and Mode 9 (inter-area) have negative damping, characterizing the instability of the power system with oscillations of increasing amplitudes.

First, the locations where the control devices will be installed need to be defined, and then, the parameters of the damping controllers should be tuned using the PSO method. Participation factors are used to define the location of the PSS devices in the power system (Kundur 1994) and the difference between the poles and zeros of the POD open-loop transfer function to define the position of the TCSC-POD controller (Moura et al. 2012).

A control device will be installed for each oscillation mode of interest in order to obtain damping levels of at least $5 \%$ and at most $10 \%$ for local modes and between 10 and $20 \%$ for inter-area modes. Two case studies are considered:

- Case study A: installation of PSSs and TCSC-POD controller for additional damping of unstable oscillation modes (Modes 1, 5, 8 and 9);

- Case study B: installation of PSSs and TCSC-POD controller for additional damping of all oscillation modes.

From additional simulations not shown in this work, the factors of interest were calculated, and it was found that 


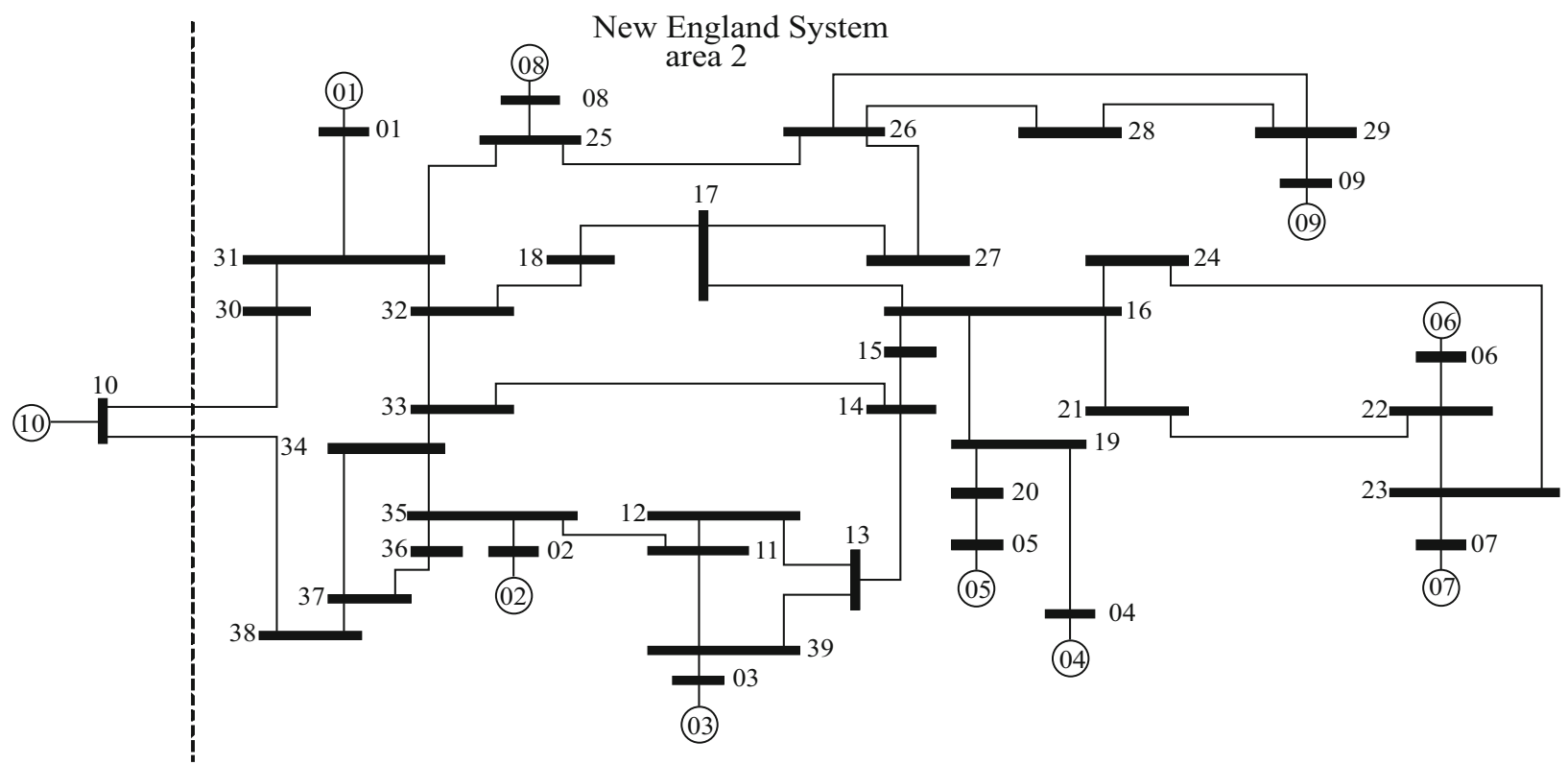

Fig. 5 Test system

Table 1 Dominant eigenvalues, damping coefficients and natural undamped frequencies (base case)

\begin{tabular}{lrrl}
\hline Modes & \multicolumn{1}{l}{ Eigenvalues } & \multicolumn{1}{l}{$\xi$} & $\omega_{n}(\mathrm{rad} / \mathrm{s})$ \\
\hline 1 & $0.0580 \pm j 6.8626$ & -0.0084 & 6.8628 \\
2 & $-0.2079 \pm j 7.2578$ & 0.0286 & 7.2608 \\
3 & $-0.1139 \pm j 6.4738$ & 0.0176 & 6.4748 \\
4 & $-0.1956 \pm j 8.2569$ & 0.0237 & 8.2592 \\
5 & $0.1693 \pm j 5.9126$ & -0.0286 & 5.9151 \\
6 & $-0.2694 \pm j 8.0989$ & 0.0332 & 8.1033 \\
7 & $-0.2432 \pm j 8.3145$ & 0.0292 & 8.3181 \\
8 & $0.0901 \pm j 6.3175$ & -0.0143 & 6.3182 \\
9 & $0.0015 \pm j 3.5348$ & -0.0004 & 3.5348 \\
\hline
\end{tabular}

Generators 1, 5 and 9 (G1, G5 and G9) are more influential in local Modes 1, 5 and 8. Analyzing the open-loop transfer function of the POD controller, the transmission line between Buses 30 and 31 (transmission line 30-31) has a greater distance between the pole of interest and its respective zero, which implies a better performance range for the POD controller (Moura et al. 2012).

Considering case study A, PSSs are installed at Generators G1, G5 and G9, while the TCSC-POD controller is installed between Buses 30 and 31. A $10 \%$ compensation of the reactance of the transmission line where the TCSC-POD controller is installed is also imposed.

The configuration given in Table 2 was obtained after running of the PSO algorithm (the results were processed in about 30 seconds with eight iterations of the algorithm).
Table 2 Configuration of the PSO with the inclusion of the PSSs (G1, G5 and G9) and the TCSC-POD in LT 30-31

\begin{tabular}{llll}
\hline Device & $T_{1}=T_{3}$ & $T_{2}=T_{4}$ & $K$ \\
\hline PSS G1 & 1.2363 & 0.0346 & 4.6534 \\
PSS G5 & 0.2915 & 0.0611 & 3.8878 \\
PSS G9 & 0.1669 & 0.0548 & 6.7013 \\
TCSC-POD & 0.0765 & 0.3704 & 0.2532 \\
\hline
\end{tabular}

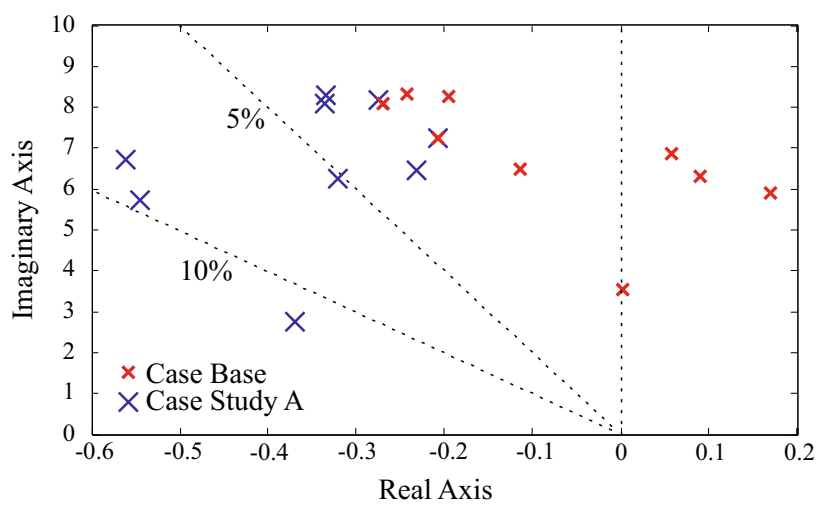

Fig. 6 Case study A

On adjusting the parameters of the PSSs and TCSC-POD controller with the data presented in Table 2, it is possible to compare the behavior of the power system with and without the supplementary damping controllers. Figure 6 shows the displacement of poles caused by the inclusion of PSSs and the TCSC-POD controller using the parameters obtained by the PSO algorithm. 
Table 3 Configuration of PSO with the performance of the PSSs (G1, G2, G3, G4, G5, G7, G8 and G9) and TCSC-POD in LT 30-31

\begin{tabular}{llll}
\hline Device & $T_{1}=T_{3}$ & $T_{2}=T_{4}$ & $K$ \\
\hline PSS G1 & 1.1321 & 0.0639 & 2.2108 \\
PSS G2 & 1.2010 & 0.0912 & 0.6431 \\
PSS G3 & 1.3963 & 0.0276 & 0.6103 \\
PSS G4 & 1.4185 & 0.0517 & 0.5700 \\
PSS G5 & 0.2861 & 0.0243 & 3.3749 \\
PSS G7 & 0.3940 & 0.0743 & 0.5633 \\
PSS G8 & 1.4707 & 0.0436 & 0.7482 \\
PSS G9 & 0.2501 & 0.0567 & 4.6833 \\
TCSC-POD & 0.0877 & 0.3011 & 0.1321 \\
\hline
\end{tabular}

Figure 6 shows that the oscillation modes of the base case (in red) are displaced compared to those of case study A (in blue). The unstable local modes are shifted to the area with damping between 5 and $10 \%$, whereas the unstable interarea mode is shifted to the area with damping between 10 and $20 \%$. These values are considered for the safety margin in the operation of the power system.

Moreover, this figure shows that five other local modes continue with damping below $5 \%$ and the installation of other PSSs is considered for additional damping to these modes.

The factors of participation were again verified in order to define the best locations to install the other PSSs. Besides the PSSs and TCSC-POD controller installed in the case study, another five PSSs were installed at Generators 2, 3, 4, 7 and 8 (G2, G3, G4, G7 and G8).

Therefore, the system will operate with eight PSSs (installed in G1, G2, G3, G4, G5, G7, G8 and G9) and the TCSC-POD controller installed on transmission line 30-31 with this situation being treated as case study $\mathrm{B}$. The values obtained for gain and the time constants using the PSO algorithm (processing approximately 88 seconds with 26 iterations) for the PSSs and TCSC-POD controller are given in Table 3.

Figure 7 shows the displacement of oscillation modes in the base case (in red) compared to case study B (in black) after reconfiguring the system with the PSSs and TCSC-POD controller using the parameters tuned according to Table 3 . The configuration calculated by the PSO algorithm provided the desired damping (minimum of $5 \%$ for local and minimum of $10 \%$ for inter-area modes) to all oscillation modes of the power system.

Figure 8 compares the damping coefficients of the oscillation modes between the base case, case study A and case study B. The lack of damping to oscillation modes is evident in the base case, in particular in respect of the oscillation modes that have negative damping coefficients. The algorithm calculated configurations to provide damping as

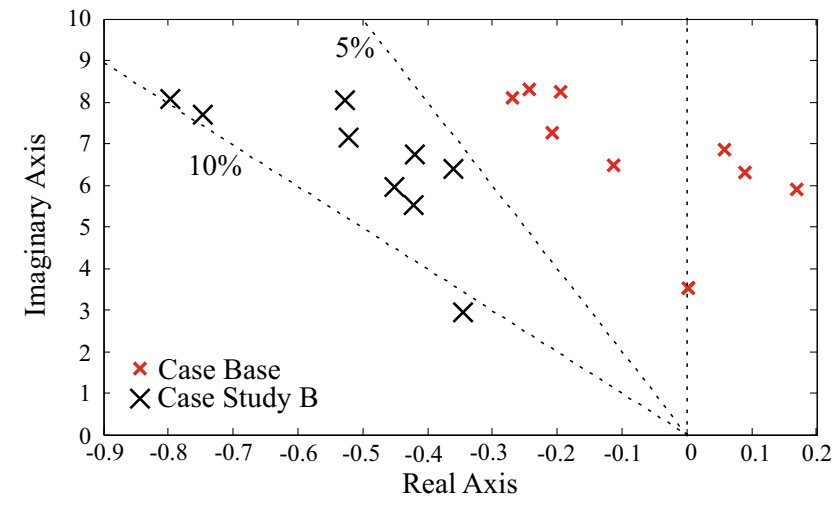

Fig. 7 Case study B

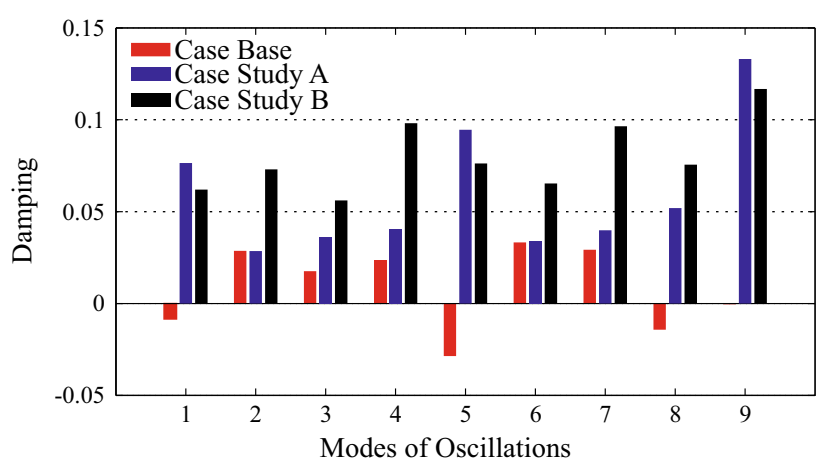

Fig. 8 Comparison of damping coefficients for the case studies

defined by the project $0.05 \leq \xi_{w i} \leq 0.1$ for local modes and $0.1 \leq \xi_{w i} \leq 0.2$ for inter-area modes in both case study A and case study B.

The oscillation Modes 1, 5 and 9 of case study A have higher damping coefficients compared to case study B. This is due to the independence of one case with the other where the settings were independently found by the PSO algorithm. In contrast, the overall damping of the system has an operating safety margin in case study B, which is not necessarily the situation in case study A.

Two distinct situations are considered to check the behavior of the power system with load variations on the buses: increases and decreases of $5 \%$ in the active and reactive powers. Figure 9 shows the damping coefficients of each oscillation mode in case study B. It can be seen that no oscillation mode was damped below the desired level ( $5 \%$ for local modes and $10 \%$ for inter-area modes). This situation was repeated in several loading situations; however, not all loading situations were tested by this algorithm, and so an optimal response cannot be guaranteed in all power system operating ranges.

On considering the power system without changes in the loads and a disturbance rejection of $0.05 \mathrm{pu}$. in Generator G2 (system reference generator), changes in the internal angle of Generator G5 $\left(\Delta \delta_{5}-\Delta \delta_{2}\right)$ and its dynamic behavior were 


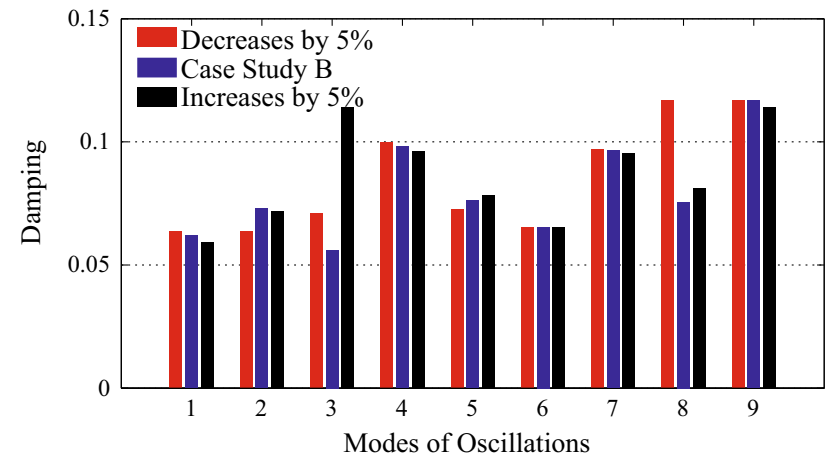

Fig. 9 Comparison of damping coefficients for case studies B

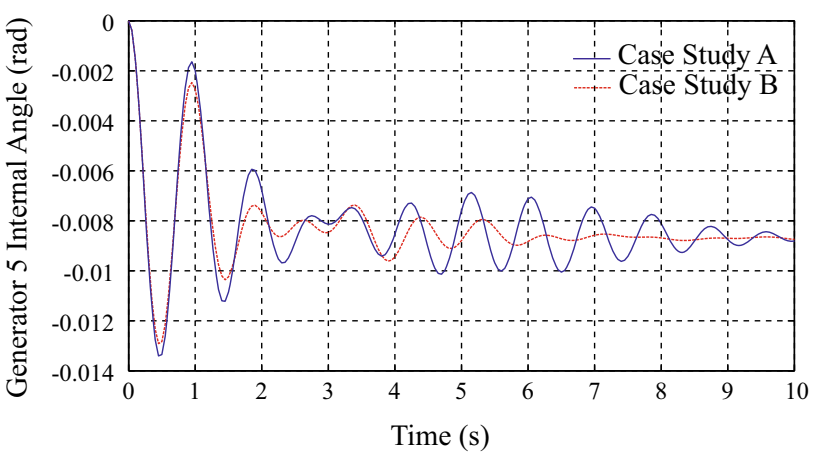

Fig. 10 Internal angle of Generator G5 with the parameters of case studies B

calculated as shown in Fig. 10. One can see that in case study B, the system tends toward the permanent regime value before case study A, which gives the power system a better performance when subjected to small perturbations.

\section{Conclusions}

The work presents discussions related to small-signal stability, considering the performance and simultaneous tuning of controllers, in order to increase damping levels of lowfrequency oscillation modes in power systems.

The efficiency of the proposed optimization algorithm will depend on the complexity of the power system. In a less complex power system, the solution using the PSO algorithm is faster compared with other published studies (Menezes et al. 2014). The same objective function was used in the work cited with just the coordinated solution search structure being different.

The PSO algorithm was used to adjust the parameters of the supplementary damping controllers. The objective function of the algorithm is to position the oscillation mode of interest in a defined region using the desired damping coefficients (5\% for local modes and $10 \%$ for inter-area modes).
Nine oscillation modes were detected in a test power system subjected to unstable operation conditions characterized by oscillations of increasing amplitude; three local modes and one inter-area mode were responsible for this instability.

Three PSSs and one TCSC-POD controller with locations and parameter settings defined by the techniques discussed in this paper were added to make the power system stable. With this configuration, the power system became stable, though there was the need to install other supplementary PSSs at five other synchronous generators to increase the power system damping levels.

With eight PSSs and one TCSC-POD controller, the damping to local oscillation modes was greater than $5 \%$ and to inter-area oscillation modes, it was greater than $10 \%$ with a good operating safety margin in relation to small-signal stability. This was confirmed by the results considering variations of active and reactive power in the power system buses.

The PSO tool is effective to tune the parameters regardless of the number of supplementary damping controllers; this is an advantage over conventional methods found in the literature. A disadvantage compared to classical methods is the time needed for simulations; however, this is not a major constraint, as the adjustment of the damping controller parameters is carried out prior to their installation in the power system.

\section{References}

Abido, M. (2002). Optimal design of power-system stabilizers using particle swarm optimization. IEEE Transactions on Energy Conversion, 17(3), 406-413. doi:10.1109/TEC.2002.801992.

Araujo, P. B., \& Zaneta, L. C. (2001). Pole placement method using the system matrix transfer function and sparsity. International Journal of Electric Power System \& Energy Systems, 23(3), 173-178.

Basler, M., \& Schaefer, R. (2008). Understanding power-system stability. IEEE Transactions on Industry Applications, 44(2), 463-474.

Bratton, D., \& Kennedy, J. (2007). Defining a standard for particle swarm optimization. In Swarm intelligence symposium-SIS, Honolulu, pp. 120-127.

Gurrala, G., \& Sen, I. (2010). Power system stabilizers design for interconnected power systems. IEEE Transactions on Power Systems, 25(2), 1042-1051. doi:10.1109/TPWRS.2009.2036778.

Hassan, L. H., Moghavvemi, M., Almurib, H. A., \& Steinmayer, O. (2013). Application of genetic algorithm in optimization of unified power flow controller parameters and its location in the power system network. International Journal of Electrical Power \& Energy Systems, 46, 89-97.

IEEE STANDARDS. (2009). IEEE recommended practice for specifying thyristor-controlled series capacitors, New York. doi:10.1109/ IEEESTD.2009.5340372.

Jabr, R., Pal, B., Martins, N., \& Ferraz, J. (2010). Robust and coordinated tuning of power system stabiliser gains using sequential linear programming. Generation, Transmission and Distribution, 4(8), 893-904. doi:10.1049/iet-gtd.2009.0669.

Kennedy, J., \& Eberhart, R. (1995). Particle swarm optimization. In IEEE international conference on neural networks, Perth, vol. 4, pp. 1942-1948. 
Kundur, P. (1994). Power system stability and control. New York: McGraw-Hill.

Mahapatra, S., \& Jha, A. (2012). PSS \& TCSC coordinated design using particle swarm optimization for power system stability analysis. In 2012 2nd International conference on power, control and embedded systems (ICPCES), pp. 1-5. doi:10.1109/ICPCES. 2012.6508094.

Meikandasivam, S., Nema, R. K., \& Jain, S. K. (2010). Performance of installed TCSC projects. In International conference on power electronics, New Delhi, pp. 1-8.

Menezes, M. M., Araujo, P. B., \& Fortes, E. V. (2014). Bacterial foraging optimization algorithm used to adjust the parameters of power system stabilizers and thyristor controlled series capacitor-power oscillation damping controller, pp. 1-6. doi:10. 1109/INDUSCON.2014.7059408.

Milano, F. (2010). Power system modelling and scripting. Berlin, Heidelberg, New York: Springer.

Molina, D., Venayagamoorthy, G., Liang, J., \& Harley, R. (2013). Intelligent local area signals based damping of power system oscillations using virtual generators and approximate dynamic programming. IEEE Transactions on Smart Grid, 4(1), 498-508. doi:10.1109/TSG.2012.2233224.

Morsali, J., Kazemzadeh, R., \& Azizian, M. (2013). Coordinated design of MPSS and TCSC-based damping controller using PSO to enhance multi-machine power system stability. In 201321 st Iranian conference on electrical engineering (ICEE), pp. 1-6.
Moura, R. F., Furini, M. A., \& Araujo, P. B. (2012). Estudo das limitações impostas ao amortecimento de oscilações eletromecânicas pelos zeros da FTMA de controladores suplementares. Controle \& Automação, 23(2), 190-201.

Rogers, G. (2000). Power system oscillations. New York: Springer.

Sen, K., \& Sen, M. (2009). Introduction to FACTS controllers. Hoboken, NJ: IEEE Press/Wiley.

Shayeghi, H., Safari, A., \& Shayanfar, H. (2010). PSS and TCSC damping controller coordinated design using PSO in multi-machine power system. Energy Conversion and Management, 51(12), 2930-2937.

Simoes, A., Savelli, D., Pellanda, P., Martins, N., \& Apkarian, P. (2009). Robust design of a TCSC oscillation damping controller in a weak $500-\mathrm{kV}$ interconnection considering multiple power flow scenarios and external disturbances. IEEE Transactions on Power Systems, 24(1), 226-236. doi:10.1109/TPWRS.2008.2006999.

Talaq, J. (2012). Optimal power system stabilizers for multi machine systems. International Journal of Electric Power System \& Energy Systems, 43(1), 793-803.

Zhang, J., Chung, C., \& Han, Y. (2012). A novel modal decomposition control and its application to PSS design for damping interarea oscillations in power systems. IEEE Transactions on Power Systems, 27(4), 2015-2025. doi:10.1109/TPWRS.2012.2188820.

Zhang, X. P., Rehtanz, C., \& Pal, B. (2006). FACTS-devices and applications. Berlin, Heidelberg: Springer. 